\section{Resultados obtenidos con dos tipos de soluciones de rehidratación oral}

La diarrea, como es bien sabido, sigue siendo una de las principales causas de mortalidad infantil en todo el mundo. Si bien hasta el decenio de los setenta el tratamiento estándar consistía en la rehidratación intravenosa, posteriormente se adoptó el uso de sales de rehidratación oral, que son particularmente útiles cuando hay escasez de soluciones inyectables o cuando los servicios de salud y los recursos humanos no son los adecuados para la administración de tales soluciones.

Durante las últimos 20 años la Organización Mundial de la Salud (OMS) ha venido recomendando una fórmula de rehidratación oral estándar que consiste en $90 \mathrm{mmol} / \mathrm{L}$ de sodio y $111 \mathrm{mmol} / \mathrm{L}$ de glucosa, a fin de conseguir una osmolaridad total de $311 \mathrm{mmol} / \mathrm{L}$. Esta combinación probablemente facilita la absorción de líquidos, ya que la glucosa, que se absorbe junto con el sodio en el intestino delgado, promueve la absorción de este último y del agua. No obstante, aún no se define claramente la concentración ideal de sodio, ya que en algunos pacientes que han recibido esta solución la natremia ha ascendido a cifras superiores a la que se considera normal para el ser humano $(150 \mathrm{mmol} / \mathrm{L})$.

Un grupo de investigadores ha llevado a cabo una interesante revisión de todos los ensayos clínicos prospectivos aleatorizados y controlados que se han publicado de 1966 a junio de 2001 para comparar los efectos en los niños de las soluciones de rehidratación oral de osmolaridad estándar (311 mmol/ L) y reducida ( $<250 \mathrm{mmol} / \mathrm{L}$ por reducción de sodio). En todos los casos los pacientes estudiados fueron niños con diarrea aguda de un máximo de 5 días de duración, y hubo un total de 41 estudios, de los cuales solamente 15 cumplían los criterios de inclusión. La estrategia de búsqueda consistió en el rastreo de las siguientes bases de datos: Medline (1966 a junio de 2001); Embase (1988 a mayo de 2001); Cochrane (segundo número, 2001); y Current Contents (junio de 2001). Las palabras clave para la búsqueda bibliográfica fueron child, diarrhoea, fluid therapy, oral rehydration, osmolar y rehydration solutions. También se examinaron las citaciones en las revisiones e informes de ensayos, y se estableció contacto son los investigadores de estudios en marcha. La principal variable de interés fue la infusión de líquidos intravenosos sin programación previa; las variables de interés secundario fueron el volumen de las heces, la presencia de vómitos y la presencia de hiponatremia.

En un metaanálisis de nueve ensayos en que se investigó la principal variable de interés, la solución de rehidratación oral de osmolaridad reducida se asoció con menos infusiones intravenosas sin programación previa, en comparación con la solución de rehidratación estándar recomendada por la OMS (RO'1: 0,61; IC95\%: 0,47 a 0,81). En tres de los ensayos ningún paciente tratado con la solución de osmolaridad reducida necesitó una infusión intravenosa. En los 13 estudios en que se estudiaron las variables de interés secundario, los niños tratados con esta solución tuvieron un menor volumen de diarrea y vómitos menos frecuentes. En los seis ensayos en que se estudió la natremia no se halló ninguna diferencia significativa entre los grupos tratados con los dos tipos de solución. (Hahn S, Kim Y, Garner P. Reduced osmolarity oral rehydration solution for treating dehydration due to diarrhoea in children: systematic review. BMJ 2001;323:81-85.)

\section{La inmunidad contra la tos ferina se debilita con el tiempo}

Según un estudio efectuado en Italia, la inmunidad contra la tos ferina que se adquiere mediante la vacunación no es permanente. Para conservarla, quizá sea necesario administrar una dosis de refuerzo de la vacuna a adolescentes y adultos.

Nicola Principi et al., de la Universidad de Milán, evaluaron a 38 niños entre los 5 y 6 años de edad que habían recibido tres dosis de vacuna combinada (antidiftérica, antitetánica, antipertúsica acelular triple y antihepatitis B [DtaP-HBV]) antes de cumplir el año de edad (a los 3, 5 y 11 meses de nacidos). La respuesta inmunitaria de estos niños se comparó con la de otros 21 niños de la misma edad que habían tenido tos ferina durante el primer año de vida y que solo habían recibido la vacuna DT-HBV.

Se midieron los títulos séricos de inmunoglobulina G (IgG) específica contra Bordetella pertussis, la proliferación de linfocitos $\mathrm{T}$ específicos contra esta bacteria en sangre periférica, y la secreción de

$1 \mathrm{RO}=$ razón de odds (odds ratio o razón de posibilidades). 
citoquinas. Se encontró que los niños vacunados y los que tenían inmunidad natural tenían un grado similar de inmunidad residual contra la tos ferina. Cinco años después de la inmunización, muy pocos niños tenían aún concentraciones importantes de IgG sérica contra cada uno de los tres antígenos de Bordetella pertussis, o una respuesta inmunitaria sostenida de tipo celular contra la bacteria.

El grupo que había sufrido la enfermedad mostró mayoritariamente una respuesta inmunitaria tipo I, mientras que el grupo vacunado mostró, en general, una respuesta tipo 2 alterada. No obstante, las diferencias cuantitativas en lo referente a la cantidad de citoquinas producida por cada grupo fueron mínimas.

Sobre la base de estos resultados, el grupo de investigación de Principi recomienda que todo niño vacunado contra la tos ferina o con antecedentes de la enfermedad reciba una dosis de refuerzo de la vacuna a fin de impedir que mengüe la respuesta inmunitaria humoral y celular contra la bacteria. (Esposito S, Agliardi T, Giammanco A, Faldella G, Cascio A, Bosis S, et al. Long-term pertussis-specific immunity after primary vaccination with a combined diphtheria, tetanus, tricomponent acellular pertussis, and hepatitis $B$ vaccine in comparison with that after natural infection. Infect Immunol 2001;69(7):4516-4520.)

\section{Profilaxis tras la exposición laboral al VIH en el siglo XXI}

Desde 1988 que se viene ofreciendo profilaxis antirretrovírica tras la exposición laboral al VIH, pero todavía persisten algunas dudas importantes que son analizadas en este artículo.

La primera dice respecto a la eficacia de la profilaxis tras la exposición (PTE). El riesgo de infección laboral tras la exposición parenteral a la sangre de un paciente infectado por el virus de la inmunodeficiencia humana (VIH) es muy bajo (aproximadamente $0,3 \%$ ), lo cual hace prácticamente imposible reunir un número de pacientes suficiente para realizar un ensayo clínico. En estas circunstancias, los datos acerca de la eficacia de la PTE provienen de tres fuentes: los estudios realizados en modelos animales, la prevención de la transmisión vertical del VIH en el ser humano y un estudio mundial retrospectivo de casos y controles.

Varias investigaciones han demostrado la eficacia de diferentes fármacos antirretrovíricos para prevenir las infecciones por retrovirus en animales $y$ en un estudio reciente realizado en macacos se ha comprobado que el éxito de la profilaxis depende tanto de la duración del tratamiento (protección en todos los animales tratados durante 28 días, en la mitad de los tratados durante 10 días y en ninguno de los tratados durante solo 3 días) como de la relación temporal entre la exposición y el inicio del tratamiento (protección del 100\% cuando el tratamiento se inició a las 24 horas, del 50\% cuando se inició a las 48 horas y del 25\% cuando se inició a las 72 horas).

En 1994 se publicó el primer estudio que demostró que el tratamiento con zidovudina administrada a la madre durante el embarazo y el parto, $\mathrm{y}$ al niño durante las primeras 6 semanas de vida, reduce la transmisión vertical del VIH en un $67 \%$. Posteriormente, esto ha sido confirmado por varios estudios en los que se han utilizado diferentes fármacos y pautas terapéuticas y en algunos de los cuales solo se trataron los niños. Estos estudios constituyen una prueba convincente de que la PTE es eficaz en el ser humano, al menos en lo que se refiere a la transmisión vertical del virus.

En el estudio de casos y controles de trabajadores sanitarios con exposición laboral al VIH, la PTE con zidovudina se asoció con una reducción del riesgo de infección superior al $80 \%$.

Otro problema consiste en el hecho de que muchas veces la prescripción de la PTE se les solicita a quienes no están muy familiarizados con los antirretrovíricos. Un error potencialmente frecuente consiste en partir del principio de que cuanto más, mejor, y prescribir un tratamiento tan complejo que acabe dificultando su cumplimiento. En la mayoría de los casos de exposición laboral bastan dos fárma$\cos \mathrm{y}$, en ausencia de datos que demuestren la eficacia de tratamientos más breves, se sigue recomendando el régimen tradicional de 28 días.

Cuando la fuente de la exposición es un paciente que ha sido tratado con múltiples fármacos antirretrovíricos, las directrices más recientes del Servicio de Salud Pública de los Estados Unidos de América recomiendan que, si hay fuerte sospecha o se ha confirmado resistencia, se añada un fármaco de una clase a la cual el paciente fuente nunca haya estado expuesto.

El embarazo no es motivo para no administrar PTE a una mujer con exposición laboral al VIH. La decisión de recibir o no la profilaxis le corresponde a la mujer, después de haber sido informada de los riesgos de infección y de los efectos adversos del tratamiento para ella y para el feto. Si opta por el tratamiento, se le debe ofrecer el régimen con mayores probabilidades de prevenir la infección y se debe observar cuidadosamente su evolución para identificar rápidamente cualquier acontecimiento adverso.

En resumen, la PTE en trabajadores sanitarios con exposición laboral al VIH debe seguir cuatro 
principios básicos: 1) proporcionar acceso inmediato al tratamiento; 2 ) asegurarse de que se ha producido una exposición antes de administrar la PTE; 3) seleccionar un régimen que el trabajador pueda cumplir, teniendo en cuenta el tratamiento y la carga vírica del paciente que ha originado la exposición, y 4) vigilar cuidadosamente la posible aparición de efectos adversos del tratamiento y, de ser posible, tratarlos de forma preventiva. (Henderson DK. HIV postexposure prophylaxis in the 21st century. Emerg Infect Dis 2001;7:254-258.)

\section{El uso del chupete, el destete precoz y el llanto}

Tanto los clínicos como los especialistas en salud pública han manifestado su preocupación por el hecho de que el uso del chupete cause "confusión con el pezón" y, por consiguiente, lleve al destete precoz. Tanto es así que uno de los objetivos de una iniciativa conjunta de la Organización Mundial de la Salud y el Fondo de las Naciones Unidas para la Infancia consiste en evitar el uso del chupete. De hecho, varios estudios observacionales han descrito una fuerte asociación entre el uso del chupete y el destete precoz, pero este tipo de estudios no permite establecer una relación de causalidad.

Los objetivos de este ensayo clínico aleatorio controlado con doble enmascaramiento consistieron en determinar: 1) si la sustitución del uso del chupete por otras medidas para calmar al lactante reduce el riesgo de destete precoz y aumenta la frecuencia o duración del llanto, y 2) si se obtienen resultados distintos cuando los datos son analizados en función de la asignación aleatoria y cuando son analizados como si se tratara de un estudio observacional, sin tener en cuenta dicha asignación.

El estudio se realizó entre enero de 1998 y agosto de 1999 en un hospital universitario de Quebec, Canadá. Las participantes fueron mujeres con partos no gemelares de niños con un mínimo de 37 semanas de gestación y $2500 \mathrm{~g}$ de peso al nacer que tenían proyectado darles de mamar a sus hijos al menos hasta los 3 meses. Se estimó que una reducción del uso del chupete del 60 al 40\% reduciría el riesgo de destete precoz (antes de los 3 meses) del 40 al $35 \%$ y que, con un nivel $\alpha$ de 0,05 y un nivel $\beta$ de 0,10 , serían necesarios aproximadamente 140 lactantes por grupo.

Las participantes fueron asignadas aleatoriamente a una de dos intervenciones que consistieron en una entrevista con una enfermera en la que se les proporcionó información general sobre la lactancia materna. La única diferencia entre el grupo experimental $(n=140)$ y el grupo de control $(n=141)$ radicó en el hecho de que al primero se le recomendó específicamente evitar el uso del chupete y recurrir a otras medidas para calmar al lactante. Las madres rellenaron un cuestionario sobre el comportamiento de los lactantes en 3 días consecutivos a las 4, 6 y 9 semanas. Además, a los 3 meses fueron entrevistadas por un asistente que desconocía el grupo al que habían sido asignadas. La variable primaria de eficacia fue el destete precoz. Como variables secundarias se registraron la frecuencia (número de episodios diarios) y duración total (minutos al día) de los episodios de llanto.

El estudio fue completado por 258 de las 281 mujeres (91,8\%): 127 del grupo experimental y 131 del grupo de control. La intervención experimental aumentó la evitación del uso del chupete (del 16,0\% en el grupo de control al 38,6\% en el grupo experimental), redujo su uso diario (55,7\% frente a 40,8\%, respectivamente) y redujo el número de inserciones diarias del chupete ( 2,4 frente a 0,8 a las 4 semanas; 3,0 frente a 0,8 a las 6 semanas, y 3,0 frente a 1,3 a las 9 semanas). En el análisis basado en la asignación aleatoria, la intervención experimental no tuvo efectos detectables sobre el destete a los 3 meses $(18,9 \%$ en el grupo experimental frente a $18,3 \%$ en el grupo de control; riesgo relativo: 1,0; intervalo de confianza del 95\%: 0,6 a 1,7) ni sobre el llanto (duración total diaria de 143 frente a 151 minutos a las 4 semanas, 128 frente a 131 minutos a las 6 semanas y 110 frente a 104 minutos a las 9 semanas, en los grupos experimental y de control, respectivamente). Sin embargo, cuando los datos fueron analizados como si se tratara de un estudio observacional, se registró una fuerte asociación entre la exposición al uso diario del chupete y el destete a los 3 meses (25,0\% en el grupo expuesto frente a $12,9 \%$ en el no expuesto; riesgo relativo: 1,9; intervalo de confianza del 95\%: 1,1 a 3,3.)

La intervención tuvo un éxito considerable en lo que se refiere a la reducción del uso del chupete, aunque esto no influyó sobre el llanto a las 4, a las 6 ni a las 9 semanas, ni tampoco sobre el riesgo de destete precoz cuando los datos fueron analizados teniendo en cuenta la asignación aleatoria. En cambio, cuando fueron analizados como si se tratara de un estudio observacional, sí hubo una significativa asociación entre el uso del chupete y el destete antes de los 3 meses. Estos resultados indican que el uso del chupete es un marcador de dificultades con la lactancia materna o de una escasa motivación para seguir con este tipo de alimentación, más que una verdadera causa de destete precoz. Aunque estos resultados requieren confirmación, la ausencia de una relación causal entre el uso del chupete y el destete precoz debería llevar a los organismos internacionales a reexaminar su oposición al uso del chupete. (Kramer MS, Barr RG, Dagenais S, 
Yang $\mathrm{H}$, Jones $\mathrm{P}$, Ciofani L, et al. Pacifier use, early weaning, and cry/fuss behavior. A randomized controlled trial. JAMA 2001;286:322-326.)

\section{Diarreas infecciosas: directrices prácticas}

Estas directrices de la Sociedad Estadounidense de Enfermedades Infecciosas (Infectious Diseases Society of America) tienen como objetivo proporcionar un documento de consenso acerca de las pruebas diagnósticas, de los tratamientos y de las medidas de salud pública que se deben adoptar ante los casos de diarrea de origen infeccioso. Las directrices han sido elaboradas por un grupo de internistas, pediatras, salubristas y microbiólogos de reconocido prestigio en el campo de las enfermedades infecciosas intestinales, están dirigidas fundamentalmente a los países industrializados, y en particular a los Estados Unidos de América, y, siempre que posible, proporcionan información sobre la calidad de las pruebas disponibles (I: recomendación basada en al menos un ensayo clínico aleatorizado controlado; II: recomendación basada en al menos un ensayo clínico no aleatorizado, un estudio de cohortes o de casos y controles o múltiples series de pacientes, o III: recomendación basada en la opinión de expertos) y la solidez de las pruebas (A: buenas pruebas a favor del uso; B: pruebas razonables a favor del uso; C: pruebas escasas a favor o en contra del uso; D: pruebas razonables en contra del uso, o E: buenas pruebas en contra del uso).

La diarrea se define como un aumento del contenido líquido, volumen o frecuencia de las deposiciones; la diarrea infecciosa como la causada por algún agente infeccioso, a menudo acompañada de náuseas, vómitos o cólicos; la diarrea aguda como la de duración $\leq 14$ días, y la diarrea persistente como la de duración > 14 días.

Las recomendaciones se dividen en dos grupos: las destinadas a la práctica clínica y las centradas en la salud pública.

Los principales riesgos de la diarrea son la deshidratación y, en los países en desarrollo, la malnutrición. Por consiguiente, el tratamiento inicial debe incluir la rehidratación (A-I). Las soluciones de rehidratación oral recomendadas por la Organización Mundial de la Salud se pueden preparar mezclando 3,5 g de $\mathrm{NaCl}, 2,5 \mathrm{~g}$ de $\mathrm{NaHCO}_{3}$ (o 2,9 g de citrato sódico), $1,5 \mathrm{~g}$ de $\mathrm{KCl}$ y $20 \mathrm{~g}$ de glucosa o de un polímero de glucosa (por ejemplo, 4 cucharadas de azúcar o 50 a $60 \mathrm{~g}$ de harina de cereales) por litro de agua. De este modo se obtiene una solución con aproximadamente $90 \mathrm{mM}$ de $\mathrm{Na}, 20 \mathrm{mM}$ de $\mathrm{K}$, $80 \mathrm{mM}$ de $\mathrm{Cl}, 30 \mathrm{mM}$ de $\mathrm{HCO}_{3}$ y $111 \mathrm{mM}$ de glucosa. La rehidratación oral no solo es más barata y me-nos dolorosa que la rehidratación intravenosa, sino también más segura, pues evita el riesgo de sobrehidratación. También se puede considerar la necesidad de administrar suplementos de vitamina A y cinc en caso de déficit. En la actualidad se están investigando nuevas medidas de rehidratación oral y nutrición a base de la administración de glutamina o sus derivados, que ayudan a reparar las lesiones de la mucosa.

El examen del paciente requiere en primer lugar una cuidadosa anamnesis clínica (A-II) (comienzo de la enfermedad, duración de los síntomas, características de las heces, frecuencia y volumen de las defecaciones, presencia de síntomas disentéricos y de deshidratación y otros síntomas) y epidemiológica (viajes a países en desarrollo, ingreso o trabajo en centros de día, consumo de alimentos poco seguros, contacto con animales con diarrea, otras personas enfermas en el entorno del paciente, medicaciones habituales, enfermedades predisponentes, relaciones sexuales anales y trabajo como manipulador de alimentos). En el examen físico hay que prestar especial atención a las constantes vitales (fiebre, frecuencia cardíaca y tensión arterial) a los signos de deshidratación, al dolor abdominal y a las alteraciones de la consciencia.

Toda diarrea de más de 1 día de duración requiere un examen de heces, particularmente si se acompaña de fiebre, heces sanguinolentas, enfermedad sistémica, uso reciente de antibióticos, hospitalización o deshidratación. Además, pueden ser necesarias otras pruebas complementarias (bioquímica, hemograma, hemocultivo, análisis de orina, radiografía abdominal, anoscopia o endoscopia).

Los exámenes de heces deben ser realizados de forma selectiva, dependiendo de que se trate de una diarrea extrahospitalaria o del viajero, especialmente si se acompaña de fiebre o heces sanguinolentas, de una diarrea hospitalaria que comienza más de 3 días después del ingreso, o de una diarrea persistente (B-II). Los pacientes hospitalizados requieren pruebas de detección de toxinas de Clostridium difficile, especialmente si tienen dolor abdominal. Toda diarrea de más de 7 días de duración requiere exámenes de heces. Ante la sospecha de un brote de gastroenteritis pueden ser necesarios estudios especiales y aislamiento de Escherichia coli. Aunque se están desarrollando nuevas pruebas diagnósticas muy prometedoras basadas en análisis inmunoenzimáticos y sondas de $\mathrm{ADN}$, los cultivos seguirán siendo imprescindibles para investigar la sensibilidad a los antibióticos e identificar los serotipos y subtipos en los brotes.

Cuando no se haya identificado ningún patógeno tras una investigación exhaustiva, es necesario considerar posibles causas no infecciosas o extrain- 
testinales de diarrea, tales como síndrome del intestino irritable, isquemia intestinal, abuso de laxantes, obstrucción intestinal parcial, absceso rectosigmoideo, enfermedad de Whipple, anemia perniciosa, diabetes, malabsorción, diverticulosis del intestino delgado, esclerodermia o enfermedad celíaca.

La necesidad de un tratamiento con antibióticos debe ser ponderada con cautela, sobre todo teniendo en cuenta el riesgo creciente de infección por microorganismos resistentes a los antibióticos, la sobreinfección por erradicación de la flora intestinal normal durante el tratamiento con antibióticos y la posibilidad de que los antibióticos induzcan fagos patógenos, como ocurre con el fago de la toxina de Shiga inducido por las quinolonas. En las diarreas del viajero se suele recomendar el tratamiento empírico con antibióticos debido a la probabilidad de que se deban a E. coli enterotoxígena o a otros patógenos bacterianos. El tratamiento empírico también se puede considerar en caso de diarrea de duración superior a 10 a 14 días, debido a la probabilidad de que se trate de una giardiasis, y en pacientes con diarrea febril, especialmente cuando se crea que tienen enfermedad invasora moderada o grave. Dicho tratamiento empírico puede comenzar con una quinolona, o con trimetoprima-sulfametoxazol en los niños. No obstante, hay una preocupación creciente por la resistencia de Campylobacter yeyuni a las quinolonas, caso en el que la diarrea puede empeorar debido a la erradicación de la flora normal por el antibiótico; en estos casos, la eritromicina puede reducir la duración de la enfermedad. También es preocupante la aparición de salmonelas resistentes a múltiples antibióticos, entre ellos las quinolonas. Las infecciones por E. coli productora de toxina de Shiga (STEC) no deben ser tratadas con inhibidores de la motilidad intestinal. El tratamiento antibiótico de las infecciones por STEC O157 puede incrementar el riesgo de síndrome hemolítico urémico. La vacunación parenteral (Vi) u oral (Ty21a) frente a la fiebre tifoidea es recomendable para quienes vayan a viajar a zonas endémicas.

Debido a la tardanza en la obtención de los resultados de los cultivos y de los exámenes de parásitos y huevos en las heces y al carácter autolimitado de la mayoría de las diarreas infecciosas, estas pruebas generalmente proporcionan escasa información útil desde el punto de vista clínico y muchos médicos las consideran como un gasto inútil. No obstante, pueden ser extremadamente útiles desde el punto de vista de la salud pública. La realización de pruebas diagnósticas en muestras de heces también puede estar indicada en grupos de individuos no enfermos, como los manipuladores de alimentos y el personal sanitario que atiende directamente a los pacientes. La subtipificación de los aislados es fun- damental para la detección, control y prevención de brotes. Con este fin se ha creado en los EE. UU. una red nacional de laboratorios (PulseNet) que determinan de forma rutinaria el serotipo de los aislados de STEC O157, Salmonella, Shigella y Listeria.

La notificación de las sospechas de brotes de diarrea infecciosa y de las enferemedades de declaración obligatoria es fundamental para la vigilancia de salud pública, la identificación de brotes y las medidas de prevención y control.

Otra importante medida de salud pública consiste en la educación de los pacientes, una vez que muchas diarreas de causa infecciosa pueden ser evitadas con medidas simples de higiene personal y de manipulación correcta de los alimentos. (Guerrant RL, Van Gilder T, Steiner TS, Thielman NM, Slutsker L, Tauxe RV, et al. Practice guidelines for the management of infectious diarrhea. Clin Infect Dis 2001;32:331-350.)

\section{Ausencia de efectos de los suplementos de cinc sobre la malaria}

El déficit de cinc es frecuente en los niños de los países en desarrollo y se asocia con un aumento de la susceptibilidad a varias infecciones debido a sus efectos sobre el sistema inmunitario. Varios estudios realizados sobre todo en Asia y América Latina han revelado que los suplementos de cinc tienen efectos terapéuticos y profilácticos frente a la diarrea aguda y crónica, a la disentería y a la neumonía. Dos estudios han revelado también su posible eficacia frente a la malaria. En este ensayo clínico aleatorizado doblemente enmascarado, y controlado con placebo se investiga la eficacia de los suplementos de cinc para reducir la morbilidad de la malaria por Plasmodium falciparum en niños africanos.

El estudio se realizó entre junio y diciembre de 1999 en la región noroccidental de Burkina Fasso, donde la malaria constituye una importante causa de mortalidad infantil. Los participantes fueron niños de 6 a 31 meses que residían permanentemente en la zona. La selección se hizo por lotería. Se excluyeron los niños con enfermedades subyacentes graves y del análisis final se excluyeron aquellos que habían estado ausentes de la zona durante más de 14 días consecutivos. Para detectar una reducción del $20 \%$ en el número de episodios de malaria por P. falciparum con una potencia del $90 \%$ y un nivel $\alpha$ de 0,05 , se calculó que sería necesaria una muestra de 720 niños. Aleatoriamente, los niños recibieron $12,5 \mathrm{mg}$ de sulfato de cinc o placebo todos los días, excepto los domingos, durante 6 meses. Fueron observados diariamente por un auxiliar sanitario que tomó su temperatura axilar y anotó los 
síntomas descritos por los padres. Cuando la temperatura fue $\geq 37,5^{\circ} \mathrm{C}$ se obtuvieron muestras de sangre para examen de parásitos. A los 0,3 y 6 meses se efectuaron exámenes médicos en los que también se obtuvieron muestras de sangre.

El análisis se realizó por intención de tratar. La medida de eficacia primaria fue la incidencia de episodios clínicos de malaria por P. falciparum, en presencia o en ausencia de $P$. ovale o $P$. malariae. Los episodios se definieron como una temperatura $\geq 37,5^{\circ} \mathrm{C}$ con al menos 5000 parásitos/ $\mu \mathrm{L}$ en ausencia de otras causas evidentes de fiebre. Las medidas de eficacia secundarias fueron la duración y gravedad de los episodios de malaria por P. falciparum, la prevalencia de otras causas de morbilidad, en particular diarreas, fiebre y tos, y la mortalidad por todas las causas. Se calcularon los riesgos relativos (RR), sus intervalos de confianza del 95\% (IC95\%) y los valores $P$. Mediante un modelo de regresión de Poisson se analizó el número de episodios de malaria en función del tratamiento, con y sin ajuste para las covariables edad, peso, talla, sexo y grupo étnico. Para analizar el efecto del tratamiento sobre la mortalidad se utilizó un modelo de regresión de riesgos instantáneos proporcionales, con y sin ajuste en función de las covariables.

De los 713 niños elegibles, 709 fueron asignados aleatoriamente a uno de los dos grupos: 356 al tratado con cinc y 353 al tratado con placebo. En total, 661 de 685 niños (96\%) fueron sometidos a los tres exámenes médicos. Los dos grupos eran similares con respecto a la edad y origen étnico. La prevalencia de malnutrición fue elevada (36\%). Se obtuvieron datos parasitológicos en 2065 de los 2324 (89\%) episodios febriles. El número medio mensual de episodios de malaria por $P$. falciparum fue de 0,28 con densidad parasitaria $\geq 5000 / \mu \mathrm{L}$ y 0,02 con densidad parasitaria $\geq 100000 / \mu \mathrm{L}$, sin que se ob- servaran diferencias entre los dos grupos: $\mathrm{RR}=0,98$ (IC95\%: 0,86 a 1,$11 ; P=0,77$ ) con una densidad parasitaria $\geq 5000 / \mu \mathrm{L}$ y $\mathrm{RR}=1,00$ (IC95\%: 0,64 a 1,$60 ; P=0,91)$ con una densidad parasitaria $\geq 100000 / \mu \mathrm{L}$. Tampoco hubo diferencias entre los grupos con respecto a la temperatura media, a la densidad parasitaria media, al número de días con fiebre ( $R R=1,01$; IC95\%: 0,95 a 1,07; $P=0,62) \mathrm{ni}$ al número de días con tos (RR = 1,05; IC95\%: 0,97 a $1,15 ; P=0,22)$. En cambio, el número de días con diarrea fue significativamente menor en el grupo tratado con cinc ( $R R=0,87$; IC95\%: 0,79 a 0,95; $P=0,002)$. El número de niños fallecidos fue mayor en el grupo tratado con placebo que en el tratado con cinc (12 frente a 5), aunque la diferencia no fue estadísticamente significativa. En el análisis de supervivencia con el modelo de riesgos instantáneos proporcionales se obtuvo un RR de 0,41 (IC95\%: 0,15 a 1,$19 ; P=0,1$ ), que no cambió de forma apreciable tras el ajuste en función de las covariables $(\mathrm{RR}=0,47)$.

Estos resultados no muestran que los suplementos de cinc sean eficaces frente a la malaria por $P$. falciparum, contradiciendo así los resultados de dos estudios anteriores realizados en Gambia $(n=$ 110) y Papua Nueva Guinea $(n=274)$. En cambio, se observó que el cinc es eficaz para reducir la morbilidad por diarrea y que podría contribuir a reducir la mortalidad por todas las causas. No obstante, como el estudio no fue diseñado específicamente para investigar los efectos del cinc sobre otras causas de morbilidad, esos resultados deben ser interpretados con cautela. (Müller $\mathrm{O}$, Becher $\mathrm{H}$, van Zweeden AB, Ye Y, Diallo D, Konate A, et al. Effect of cinc supplementation on malaria and other causes of morbidity in west African children: randomised double blind placebo controlled trial. BMJ 2001;322(7302):1567-1570.) 